\begin{tabular}{ll} 
IJEMR & International Journal of \\
\hline & Emerging Multidisciplinary Research
\end{tabular}

\title{
Derivation of accident reduction measures through analysis of bicycle accident occurrences: Centering on Gyeonggi-do Province
}

\author{
Byeong Kwan, Jeon', Jong Kyu, Ko², Gouzhong, $\mathrm{Li}^{3}$ * \\ ${ }^{1-2}$ Business Administration, Sunmoon University, Asan 31460, South Korea \\ ${ }^{3}$ Dept. of Management Science and Information System, Kunming University of Science and Technology, \\ 650093, China
}

\begin{abstract}
Background/Objectives: In this study, through big data analysis, the current situation and cause are identified and a plan to reduce accidents in bicycle accidents is derived. Methods/Statistical analysis: In order to secure data on bicycle traffic accidents, data from Gyeonggi-do were collected and analyzed using R and Tableau, which are big data analysis tools. After collecting the data, it was preprocessed according to the analysis. Findings: As a result of the analysis, it was found that bicycle accidents increased as the bicycle population increased. Therefore, in this study, an intersection notification and a bicycle cross-section were proposed. Improvements/Applications: The results of this study are expected to be used to develop policies that can be prevented in advance while instilling awareness of bicycle accidents.
\end{abstract}

Index Terms

Bicycle, Bicycle accident, Big data analysis, Gyeonggi-do, Accident reduction plan

\section{Corresponding author : Guozhong Li}

147508114@qq.com

- Manuscript received August 24, 2021.

- Revised September 27, 2021; Accepted October 16, 2021.

- Date of publication December 31, 2021.

(c) The Academic Society of Convergence Science Inc.

2546-1583 $\odot 2017$ IJEMR. Personal use is permitted, but republication/redistribution requires IJEMR permission. 


\section{INTRODUCTION}

Due to the spread of COVID-19, many changes are taking place due to social distancing and quarantine [1]. In particular, the number of people who use bicycles as leisure activities is increasing, and more and more people choose electric bicycles as a means of transportation for commuting and short-distance delivery [2].

These changes are expected to play an important role in the post-Corona or With Corona era.

Due to the increase in the construction of bicycle paths by local governments and policy support, the number of people enjoying bicycle riding at all ages continues to increase [3].

In addition, the introduction of public bicycles is increasing in many local governments due to successful operation and increased bicycle use [4].

The National Leisure Activity Survey was conducted by the Ministry of Culture, Sports and Tourism and the Korea Culture and Tourism Institute to understand the demand and status of leisure activities of the Korean people every two years [5].

The purpose of this survey is to analyze the actual conditions of activities affecting the leisure demand of the people according to various changes in the domestic leisure environment to understand the changes in lifestyle and the quality of life.

The survey consists of content that can grasp the current status of people aged 15 or older's participation in leisure activities and national leisure behavior through leisure satisfaction analysis, and is organized to predict future changes through time series analysis with existing survey results(Fig. 1).

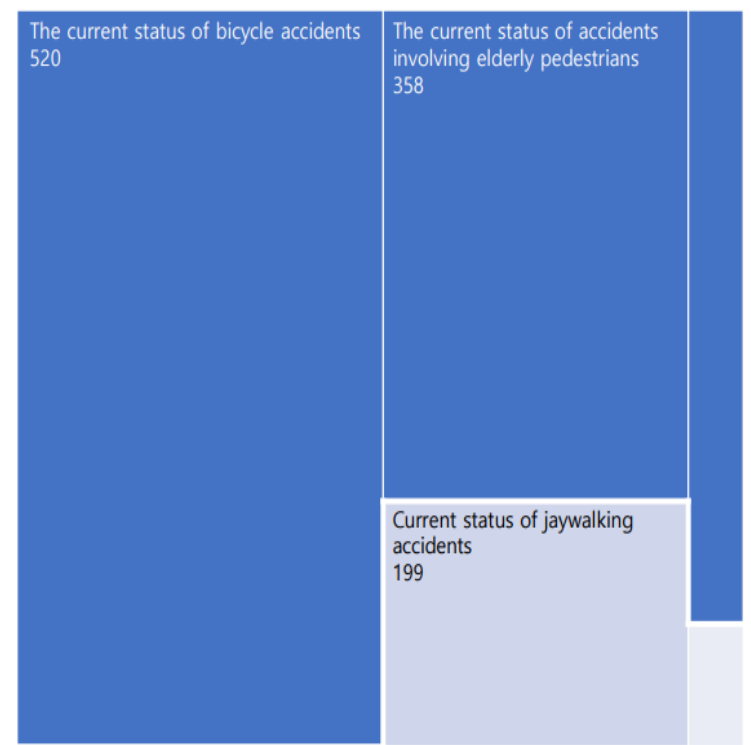

Fig. 1. Analysis of accident sites in Gyeonggi-do
According to the results of the 2018 survey, Koreans' weekday and holiday leisure hours increased to 3.3 hours and 5.3 hours, respectively, compared to 2016 , and the average monthly leisure cost was 151,000 won, up 15,000 won from 2016(Fig. 2).

Bicycle users are steadily increasing due to the increase in national leisure life, and bicycle accidents are also increasing at the same time.

Each local government designates and manages an area where accidents occur frequently for each administrative district as a multiple accident site.

However, bicycle accidents are not decreasing for various reasons.

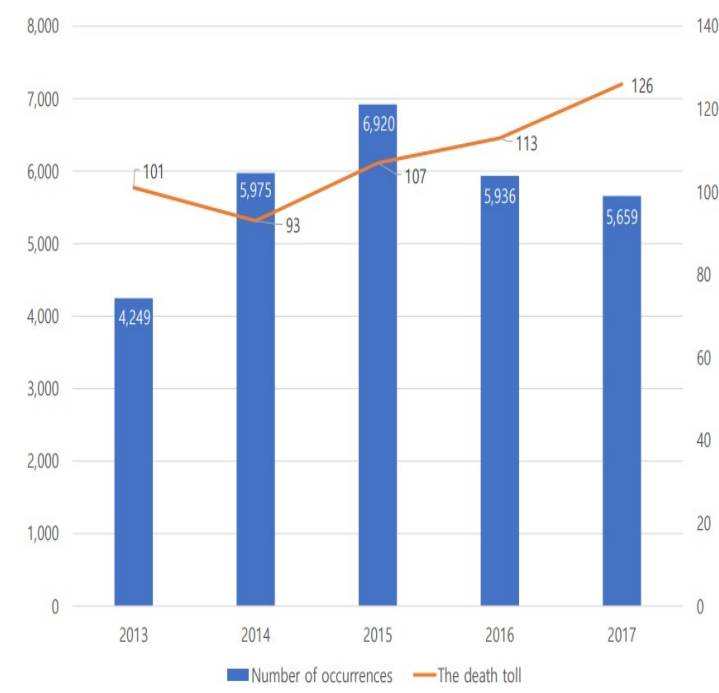

Fig. 2. Bicycle traffic accidents by year

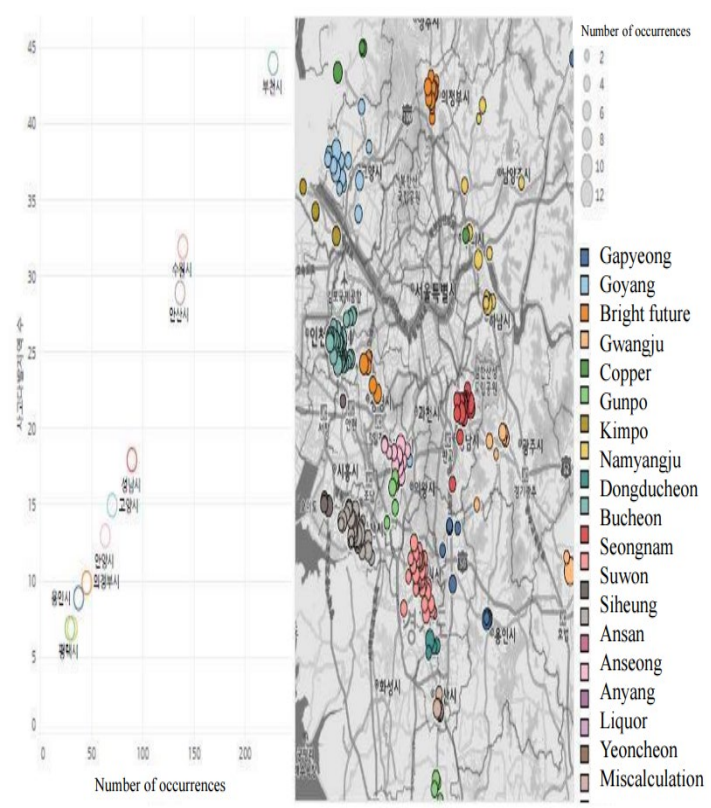




\begin{tabular}{|c|c|c|c|}
\hline \multicolumn{2}{|c|}{$\begin{array}{l}\text { The current status of bicycle } \\
\text { accidents and multiple accidents }\end{array}$} & \multicolumn{2}{|c|}{$\begin{array}{l}\text { Current status of bicycle accidents } \\
\text { per population in multiple location }\end{array}$} \\
\hline Bucheon & 131 & Bucheon & 6.454 \\
\hline Ansan & 101 & Ansan & 7.264 \\
\hline Suwon & 64 & Anyang & 13.731 \\
\hline Anyang & 42 & Osan & 13,742 \\
\hline Goyang & 31 & Gapyeong & 14.887 \\
\hline Uijeongbu & 22 & Hanam & 15.642 \\
\hline Gwangmyeong & 20 & Gwangmyeong & 18.236 \\
\hline Osan & 18 & Suwon & 19.016 \\
\hline Hanam & 16 & Uijeongbu & 19,3888 \\
\hline Siheung & 14 & Gunpo & 31.213 \\
\hline Gunpo & 11 & Goyang & 32,423 \\
\hline Namyangju & 9 & Anseong & 39.766 \\
\hline Yongin & 8 & Siheung & 40,579 \\
\hline Anseong & 8 & Seongnam & 47.215 \\
\hline Gapyeong & 5 & Guri & 47.435 \\
\hline Guri & 4 & Namyangju & 80,617 \\
\hline Gimpo & 4 & Gimpo & 98,110 \\
\hline Pyeongtaek & 4 & Pyeongtaek & 121,519 \\
\hline Hwaseong & 4 & Yongin & 124.533 \\
\hline & 4 & Hwaseong & 177.142 \\
\hline
\end{tabular}

Fig. 3. Visualization results of the accident

Therefore, this study was conducted with the aim of deriving the main accident areas of the accident site and deriving the cause of the accident, and the analysis was conducted with the aim of deriving a solution accordingly(Fig. 3).

\section{ANALYSIS METHOD}

\section{A. Data acquisition}

Prior to the analysis, the area was first limited to Gyeonggi-do to reduce the scope. Therefore, data could be obtained through Gyeonggi-do Data Dream. All available data, such as bicycle accident location data and bicycle road status, were used and preprocessed before analysis [6-9].

\section{B. Analysis Method}

$\mathrm{R}$ and Tableau, a visualization tool, were used as programs for analysis. Map visualization techniques, multiple regression analysis, etc. were used as techniques for analysis [9].

In the case of multiple regression analysis, variables were reduced through a step-by-step method to produce the initial model and build the optimal model, and the optimal model was constructed and analyzed by checking the model suitability $[10,11]$.

\section{Analysis Results}

\section{A. Visualizing the accident occurrence}

Prior to the analysis, as a result of visualizing the accident site through a map, it was possible to derive the fact that accidents occur the most in Bucheon-si, Suwon-si, etc.

In addition, as a result of visualizing the bicycle accident sites, it was possible to derive the fact that the most accidents still occur in Bucheon-si (Figure $3)$.

\section{B. Analysis of causes of bicycle traffic accidents}

Prior to the cause analysis, correlation analysis was conducted using variables such as accidents, bicycle paths, and population. As a result of the analysis, the correlation between other variables with the occurrence exceeded 0.3, showing a low correlation.

Therefore, it was attempted to examine the factors causing traffic accidents through previous studies, and as a result, it was largely divided into human factors, vehicle factors, and environmental factors, and it was derived that it occurs in combination due to several factors.

Apart from previous studies, a regression analysis was conducted focusing on facility factors to analyze the effect of facility factors on accident occurrence (Fig. 4).

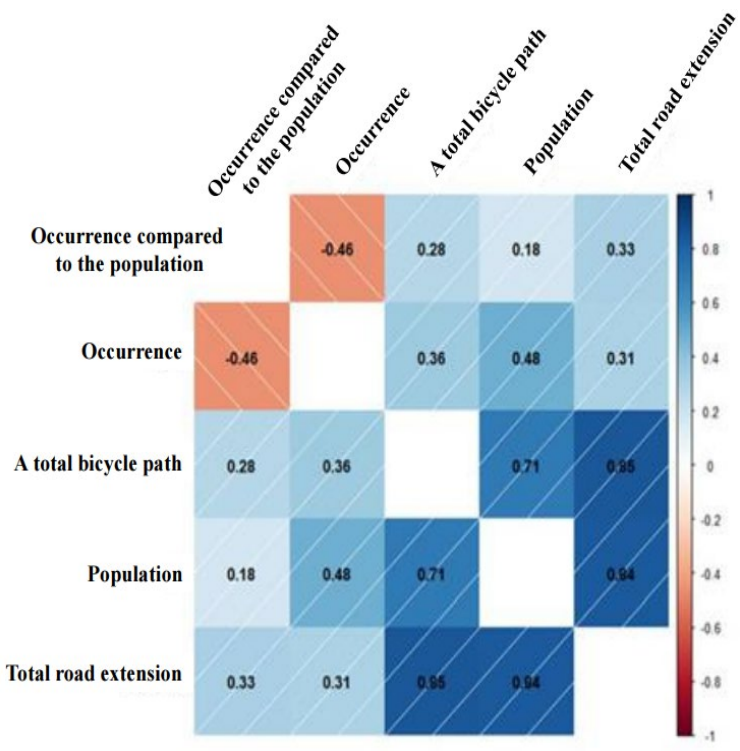

Fig. 4. Correlation analysis

\section{Deriving the number of accidents}

In order to derive information on a specific location among accident-prone areas, the address of accident-prone data was analyzed through text mining.

As a result of the analysis, the intersection was mentioned the most, and other three-way intersection, distance, and five-way intersection were mentioned. In addition, as a result of checking the accident sites of Bucheon-si and Ansan-si, the areas with the most accidents, through map visualization, it was confirmed that the accidents occur the most at the intersection. In other words, 
it was possible to derive the fact that accidents occur frequently at intersections(Fig. 5).

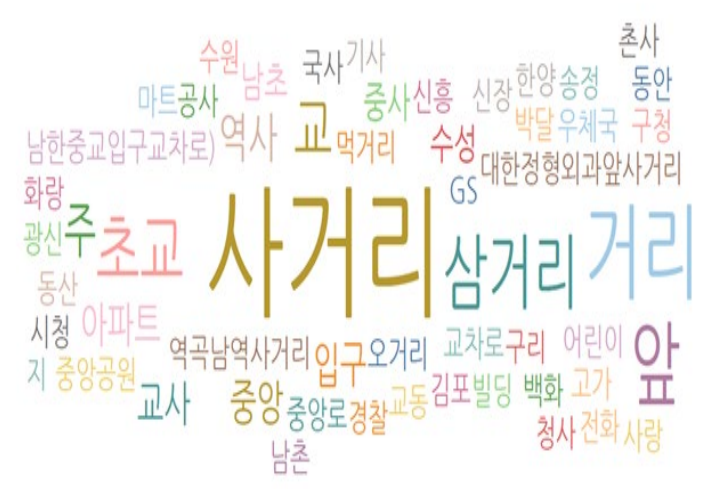

Fig. 5. Text mining results

\section{Intersection accident type}

Through previous studies, the type of accident in the intersection could be obtained, and according to him, accidents occurred the most when going straight and straight, followed by accidents during straight and left and right turns. The two cases accounted for a large proportion of $73 \%$ of the total.

As a result of checking through the actual data, when the bicycle was the perpetrator, vehicle-car accidents occurred the most, and when looking at the type of vehiclecar accidents, side collisions, that is, accidents occurred the most when each went straight. On the other hand, it was found that side collisions occur the most when bicycles are victims(Fig. 6).

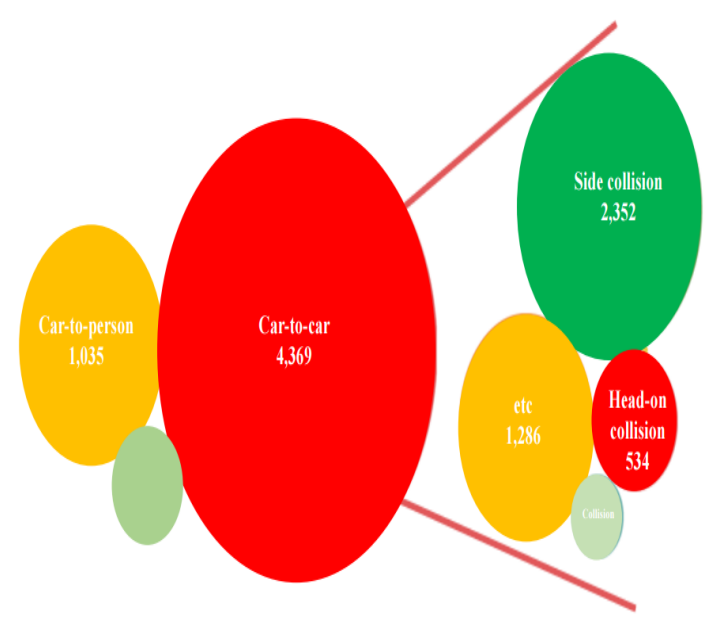

Fig. 6. Type of accident when a bicycle is the perpetrator

\section{Solution}

Through the previous analysis, it was possible to obtain results that exhibition administrative facilities rather encourage bicycle accidents, and to derive results that accidents usually occur at intersections. Based on these results, I would like to present an improvement plan to prevent accidents at intersections.

The first improvement plan is to increase the installation of "cross-notifications" that are effective in reducing actual accidents, not exhibition administrative facilities(Fig. 7).

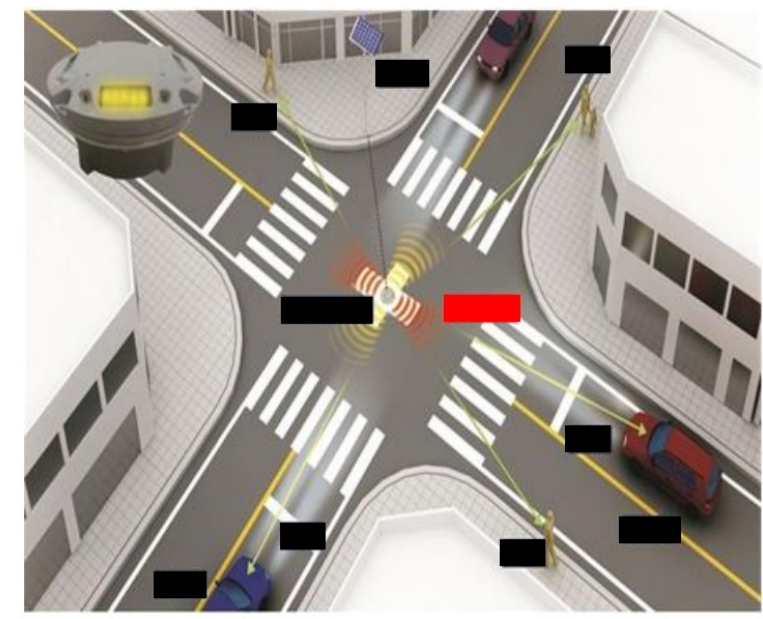

Fig. 7. Intersection notification

As a second improvement, I would like to propose a "bicycle cross-section". A bicycle crossroad is a facility that induces the driver's attention by red packaging in the conflicting section of the intersection(Fig. 8).

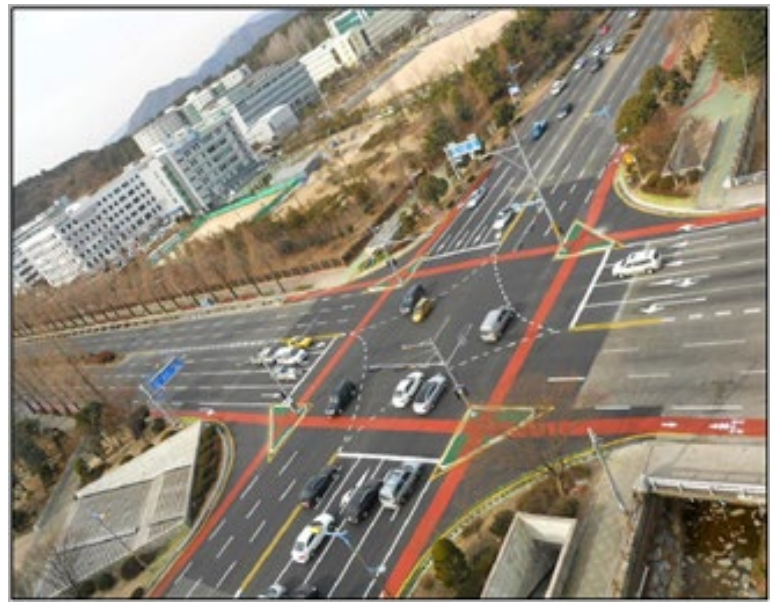

Fig. 8. Bicycle crossing

\section{Conclusion}

This analysis attempted to suggest a plan to reduce traffic accidents in bicycle accident-prone areas through correlation analysis and session analysis by extracting data from "Gyeonggi Data Dream". 
Through the analysis, it was found that existing wartime administrative structures encourage accidents, and that accidents occur at intersections.

Based on this analysis, solutions such as intersection notification and bicycle crossing could be suggested.

However, this analysis has several limitations. Due to the characteristics of the data, there are many limitations such as inconsistent periods and not reflecting the latestity. Therefore, an analysis supplemented with additional data is needed for future analysis.

\section{REFERENCES}

[1] Jiang, J., Park, E. M., \& Park, S. T. (2021). The Impact of the COVID-19 on Economic Sustainability-A Case Study of Fluctuation in Stock Prices for China and South Korea. Sustainability, 13(12), 6642.

[2] Jang, J. Y(2021). Samchuly Bicycle, the growing population, positive performance momentum https://www.asiatoday.co.kr

[3] Kang, H. S. (2008). Cycling and health. Korean Society of Civil Engineers, 57(7), 59-61.

[4] LEE, J. H., JEONG, G. O., \& SHIN, H. C. (2016). Impact analysis of weather condition and locational characteristics on the usage of public bike sharing system. Journal of Korean Society of Transportation, 34(5), 394-408.

[5] Ministry of Culture, Sports and Tourism. (2018). 2018 National Leisure Activity Survey Report.

[6] Kim, B. S., Kim, K. W., \& Park, S. T. (2015). The improvement plan for fire response time using Big data. Indian Journal of Science and Technology, 8(23), 1.

[7] Kim, K. W., Park, W. J., \& Park, S. T. (2015). A study on plan to improve illegal parking using big data. Indian Journal of Science and Technology, 8(21), 1.

[8] Hwang, J. S., Liu, C., Li, G., \& Park, S. T. (2020). A Study on Problem Solution of Culture Tourism Festival Through Big Data Analysis. International Journal of Emerging Multidisciplinary Research, 4(3), 17-21.

[9] Noh, J. H, Choi, Y. R, Choi, Y. S \& Park, S. T. (2021). Big Data Analysis to Expand Support for Cervical Cancer Vaccine. International Journal of Emerging Multidisciplinary Research, 5(3), 10-15

[10] Kim, J. W, Park, S. T \& Lee, S. W.(2018). Big data R point. Kwangmoonkag

[11] Noh, K. S, Kim, J. W, Park, S. T \& Kim, B. S.(2018). Big data analysis using R. Wowpass. 